\title{
GLOBAL SECOND DERIVATIVE ESTIMATES FOR THE SECOND BOUNDARY VALUE PROBLEM OF THE PRESCRIBED AFFINE MEAN CURVATURE AND ABREU'S EQUATIONS
}

\author{
NAM Q. LE
}

\begin{abstract}
In this paper we prove the global second derivative estimates for the second boundary value problem of the prescribed affine mean curvature equation where the affine mean curvature is only assumed to be in $L^{p}$. Our result extends previous result by Trudinger and Wang in the case of globally bounded affine mean curvature and also covers Abreu's equation.
\end{abstract}

\section{IntRoduCtion AND MAIN RESUlts}

This paper is concerned with the global second derivative estimates for the second boundary value problem of the prescribed affine mean curvature equation. This equation is a fourth order fully nonlinear partial differential equation of the form

$$
L[u]:=U^{i j} w_{i j}=f \quad \text { in } \Omega,
$$

where $u$ is a locally uniformly convex function defined in $\Omega \subset \mathbb{R}^{n}$, and throughout, $U=$ $\left(U^{i j}\right)$ is the matrix of cofactors of the Hessian matrix $D^{2} u$ of the convex function $u$, i.e.,

$$
U=\left(\operatorname{det} D^{2} u\right)\left(D^{2} u\right)^{-1}
$$

and

$$
w=\left[\operatorname{det} D^{2} u\right]^{\theta-1}, \theta<\frac{1}{n} .
$$

The second boundary value problem for (1.1) is the Dirichlet problem for the system (1.1)(1.2), that is to prescribe

$$
u=\varphi, w=\psi \text { on } \partial \Omega \text {. }
$$

The problem (1.1)-(1.3) with $\theta=\frac{1}{n+2}$ was introduced by Trudinger and Wang in their investigation of the affine Plateau problem [TW1] in affine geometry. In this context, the quantity

$$
H_{A}[u]:=-\frac{1}{n+1} L[u]
$$

Date: February 10, 2012.

2000 Mathematics Subject Classification. Primary 35J40, 35B65; Secondary 53A15.

Key words and phrases. Affine mean curvature equation, Abreu's equation, linearized Monge-Ampère equation, second boundary value problem, localization theorem. 
is the affine mean curvature of the graph of $u$ [B], NS, Si]. In particular, equation (1.1) with $f \equiv 0$ corresponds to the affine maximal surface equation [TW]. The global second derivative estimates for (1.1)-(1.3) with $\theta \in\left(0, \frac{1}{n}\right]$ were established by Trudinger and Wang in TW2] under the assumption that $f \in L^{\infty}(\Omega)$. In particular, they proved

Theorem 1.1. ([TW2, Theorem 1.2]) Let $\Omega$ be a uniformly convex domain in $\mathbb{R}^{n}$, with $\partial \Omega \in C^{3,1}, \varphi \in C^{3,1}(\bar{\Omega}), \psi \in C^{3,1}(\bar{\Omega}), \inf _{\Omega} \psi>0, f \in L^{\infty}(\Omega), f \leq 0$ and $\theta \in\left(0, \frac{1}{n}\right]$. Then, for uniformly convex solution $u \in C^{4}(\bar{\Omega})$ of (1.1)-(1.3), we have the estimate

$$
\|u\|_{W^{4, p}(\Omega)} \leq C, \text { for all } 1<p<\infty
$$

where $C$ depends on $n, p, \theta, \partial \Omega,\|f\|_{L^{\infty}(\Omega)},\|\varphi\|_{C^{4}(\bar{\Omega})},\|\psi\|_{C^{4}(\bar{\Omega})}$, and $\inf _{\Omega} \psi$.

It is very natural to ask if the estimate (1.4) holds when $f$ is only assumed to be in $L^{p}$. In this paper, we answer this question for the case $p>n$. Our first main theorem provides global derivative estimates for solutions of the prescribed affine mean curvature equation.

Theorem 1.2. Let $p>n$ and let $\Omega$ be a uniformly convex domain in $R^{n}$, with $\partial \Omega \in C^{3,1}$, $\varphi \in W^{4, p}(\Omega), \psi \in W^{2, p}(\Omega)$, inf $_{\Omega} \psi>0$ and $f \in L^{p}(\Omega), f \leq 0$. Then, for uniformly convex solution $u \in C^{4}(\bar{\Omega})$ of (1.1)-(1.3), we have the estimate

$$
\|u\|_{W^{4, p}(\Omega)} \leq C,
$$

where $C$ depends on $n, p, \theta, \partial \Omega, \Omega,\|f\|_{L^{p}(\Omega)},\|\varphi\|_{W^{4, p}(\Omega)},\|\psi\|_{W^{2, p}(\Omega)}$, and $\inf _{\Omega} \psi$.

The integrability of $f$ in Theorem 1.2 is optimal for the global estimate (1.5).

By using degree theory as in [TW2, we obtain the following result as a consequence of Theorem 1.2 .

Theorem 1.3. Let $p>n$ and let $\Omega$ be a uniformly convex domain in $R^{n}$, with $\partial \Omega \in C^{3,1}$, $\varphi \in W^{4, p}(\Omega), \psi \in W^{2, p}(\Omega), \inf _{\Omega} \psi>0$ and $f \in L^{p}(\Omega), f \leq 0$. Then, there is a unique uniformly convex solution $u \in W^{4, p}(\Omega)$ to the boundary value problem (1.1)-(1.3).

For (1.1), the condition $f \leq 0$ in the case of $\theta=\frac{1}{n+2}$ corresponds to nonnegative affine mean curvature. Our theorem also covers the case $\theta=0$ which was also treated by Zhou [Zh]. In [Zh], $f$ is bounded and $w$ is constant near the boundary $\partial \Omega$. When $\theta=0$, (1.1) is known as Abreu's equation [Ab] in the context of existence of Kähler metric of constant scalar curvature [D1, D2, D3, D4].

Our proof of Theorem 1.2 is based on

(i) the global $C^{2, \alpha}$ estimates for the Monge-Ampère equation [TW2] when the MongeAmpère measure is only assumed to be Hölder continuous, and

(ii) the global Hölder continuity estimates for solutions of the linearized MongeAmpère equations which are of independent interest.

We state these global Hölder estimates in the following theorem. 
Theorem 1.4. Let $\Omega$ be a bounded, uniformly convex domain in $\mathbb{R}^{n}$ with $\partial \Omega \in C^{3}$. Let $u: \bar{\Omega} \rightarrow \mathbb{R}, u \in C^{0,1}(\bar{\Omega}) \cap C^{2}(\Omega)$ be a convex function satisfying

$$
0<\lambda \leq \operatorname{det} D^{2} u \leq \Lambda<\infty
$$

and

$$
\left.u\right|_{\partial \Omega} \in C^{3} \text {. }
$$

Let $v$ be the continuous solution to the linearized Monge-Ampère equation

$$
U^{i j} v_{i j}=g \quad \text { in } \quad \Omega ; v=\varphi \quad \text { on } \quad \partial \Omega .
$$

Assume that

$$
\|\varphi\|_{L^{\infty}(\Omega)}+\|g\|_{L^{n}(\Omega)} \leq K
$$

and $\varphi$ is $C^{\alpha}$ on $\partial \Omega$, namely,

$$
|\varphi(x)-\varphi(y)| \leq L|x-y|^{\alpha} \text { for } x, y \in \partial \Omega .
$$

Then, $v \in C^{\beta}(\bar{\Omega})$ where $\beta$ depends only on $\lambda, \Lambda, n, K, L, \alpha$, $\operatorname{diam}(\Omega)$, and the uniform convexity of $\Omega$.

Remark 1.5. Several remarks are in order.

(1) Theorem 1.4 is the global counterpart of Caffarelli-Gutiérrez's interior Hölder estimates for solutions to the linearized Monge-Ampère equations [CG].

(2) If $g \in L^{\infty}(\Omega), \varphi \in C^{1,1}(\partial \Omega)$ and $\operatorname{det} D^{2} u \in C(\bar{\Omega})$ then Theorem 1.4 is a consequence of the global $C^{1, \alpha}$ estimates for solutions to the linearized Monge-Ampère equations obtained in [LS1]. See Theorem 2.5 and Remark 7.1 in that paper. The point of interest in Theorem 1.4 lies in the fact that the data are less regular.

Under the assumptions of Theorem 1.4, the linearized Monge-Ampère operator $L_{u}:=$ $U^{i j} \partial_{i} \partial_{j}$ is in general not uniformly elliptic, i.e., the eigenvalues of $U=\left(U^{i j}\right)$ are not necessarily bounded away from 0 and $\infty$. Moreover, $L_{u}$ can be possibly singular near the boundary; even if det $D^{2} u$ is constant in $\bar{\Omega}, U$ can blow up logarithmically at the boundary; see [LS1, Proposition 2.6]. As in [LS1, the degeneracy and singularity of $L_{u}$ are the main difficulties in proving Theorem 1.4. We handle the degeneracy of $L_{u}$ by working as in [CG with sections of solutions to the Monge-Ampère equations. These sections have the same role as Euclidean balls have in the classical theory. To overcome the singularity of $L_{u}$ near the boundary, we use a Localization Theorem at the boundary for solutions to the Monge-Ampère equations which was obtained by Savin in [S, S2].

We would like to comment on the difference between the method of the proof of Theorem 1.1 in TW2 and our method of the proof of Theorem[1.2, To prove Theorem 1.1, Trudinger and Wang first established the Lipschitz continuity of $w$ at the boundary $\partial \Omega$; precisely

$$
\left|w(x)-w\left(x_{0}\right)\right| \leq C\left|x-x_{0}\right| \forall x \in \Omega, x_{0} \in \partial \Omega .
$$

They then used this property together with convexity analysis and perturbation arguments in the spirit of Caffarelli [C2 for solutions of the Monge-Ampère equation to conclude that the boundary sections of $u$ are of good shape; that is, each of these sections lies between two 
concentric balls whose radii ratio is under control. After that, they were able to apply the arguments of Caffarelli-Gutiérrez [CG] for linearized Monge-Ampère equation to conclude that $w$ is globally Hölder continuous. Finally, the global estimates for $u$ follows from their global $C^{2, \alpha}$ estimates for solutions of Monge-Ampère equation [TW2, Theorem 1.1].

Our method of the proof of Theorem 1.2 is slightly different. When $f$ is only assumed to be in $L^{p}(p>n)$, we can only prove that $w$ is Hölder continuous at the boundary; precisely, for some $\alpha \in(0,1)$,

$$
\left|w(x)-w\left(x_{0}\right)\right| \leq C\left|x-x_{0}\right|^{\alpha} \forall x \in \Omega, x_{0} \in \partial \Omega .
$$

However, this is enough for us to establish the global Hölder continuity for $w$. To do this, we first use the Localization theorem for solutions of the Monge-Ampère equation proved by Savin [S] to obtain some mild control on the boundary sections of $u$. Precisely, each of these sections lies between two concentric balls whose radii ratio behaves like logarithm of the distance from their center to the boundary. These controls are independent of the boundary behavior of $w$. They just depend on the bounds on $w$ and information of $u$ on the boundary. The use of the Localization theorem in combination with Caffarelli-Gutiérrez's interior Hölder estimates gives us global Hölder continuity of $w$.

In this paper, we denote by $c, C, C_{0}, C_{1}, C_{2}, C_{n}$, etc... positive constant depending on structural information, such as $n, p, \theta, \partial \Omega, \Omega,\|f\|_{L^{p}(\Omega)},\|\varphi\|_{W^{4, p}(\Omega)},\|\psi\|_{W^{2, p}(\Omega)}$, and $\inf _{\Omega} \psi$ and their values may change from line to line whenever there is no possibility of confusion. We refer to such constants as universal constants.

The rest of the paper is organized as follows. In Section 2 , we establish global Hölder continuity for solutions to the linearized Monge-Ampère equations, thus proving Theorem 1.4. In Section 3, we prove Theorem 1.2 about the global second derivative estimates for the second boundary value problem of the prescribed affine mean curvature equation.

Acknowledgments. The author would like to thank Neil Trudinger for his interest, support and encouragement during the preparation of this paper. The author also acknowledges the financial support and hospitality of the Center of Mathematics and its Applications, the Australian National University, Canberra, Australia where this work was completed during his stay there as a Visiting Fellow.

\section{Global Hölder Continuity for solutions to the linearized MONGE-Ampère EQUATIONS}

In this section, we will prove Theorem 1.4. Throughout, we assume that $\Omega$ is a uniformly convex domain.

\subsection{Boundary Hölder continuity for solutions of non-uniformly elliptic equa-}

tions. The result in this subsection establishes boundary Hölder continuity for solutions to non-uniformly elliptic, linear equations without lower order terms. It is a refinement of Proposition 4.14 in [CC]; see also Lemma 4.3 in [GN1]. It states as follows. 
Proposition 2.1. Let $v$ be the continuous solution to the equation $L v:=a_{i j} v_{i j}=g$ in $\Omega$ with $v=\varphi$ on $\partial \Omega$. Here the matrix $\left(a_{i j}\right)$ is assumed to be measurable, positive definite and satisfies $\operatorname{det}\left(a_{i j}\right) \geq \lambda$. We assume that

$$
\|\varphi\|_{L^{\infty}(\Omega)}+\|g\|_{L^{n}(\Omega)} \leq K
$$

and $\varphi$ is $C^{\alpha}$ on $\partial \Omega$, namely,

$$
|\varphi(x)-\varphi(y)| \leq L|x-y|^{\alpha} \text { for } x, y \in \partial \Omega .
$$

Then, there exist $\delta, C$ depending only on $\lambda, n, K, L, \alpha$, diam $(\Omega)$, and the uniform convexity of $\Omega$ so that, for any $x_{0} \in \partial \Omega$, we have

$$
\left|v(x)-v\left(x_{0}\right)\right| \leq C\left|x-x_{0}\right|^{\frac{\alpha}{\alpha+2}}, \forall x \in \Omega \cap B_{\delta}\left(x_{0}\right) .
$$

Proof. Without loss of generality, we assume that $K>1, \lambda=1$ and

$$
\Omega \subset \mathbb{R}^{n} \cap\left\{x_{n}>0\right\}, 0 \in \partial \Omega .
$$

Take $x_{0}=0$. By the Aleksandrov-Bakelman-Pucci (ABP) estimate GT] and the assumption $\operatorname{det}\left(a_{i j}\right) \geq 1$, we have

$$
|v(x)| \leq \sup _{\Omega}\|\varphi\|_{L^{\infty}(\Omega)}+C_{n} \operatorname{diam}(\Omega)\|g\|_{L^{n}(\Omega)} \leq C_{0} K \forall x \in \Omega
$$

and hence, for any $\varepsilon \in(0,1)$

$$
|v(x)-v(0) \pm \varepsilon| \leq 3 C_{0} K:=C_{1} .
$$

Consider now the functions

$$
h_{ \pm}(x):=v(x)-v(0) \pm \varepsilon \pm C_{1}\left(\inf \left\{y_{n}: y \in \bar{\Omega} \cap \partial B_{\delta_{2}}(0)\right\}\right)^{-1} x_{n}
$$

in the region

$$
A:=\Omega \cap B_{\delta_{2}}(0)
$$

where $\delta_{2}$ is small to be chosen later.

Note that, if $x \in \partial \Omega$ with

$$
|x| \leq \delta_{1}(\varepsilon):=(\varepsilon / L)^{1 / \alpha}
$$

then, we have

$$
|v(x)-v(0)|=|\varphi(x)-\varphi(0)| \leq L|x|^{\alpha} \leq \varepsilon .
$$

It follows that, if we choose $\delta_{2} \leq \delta_{1}$ then from (2.1) and (2.2), we have

$$
h_{-} \leq 0, h_{+} \geq 0 \text { on } \partial A \text {. }
$$

On the other hand,

$$
L h_{ \pm}=g \text { in } A \text {. }
$$

The ABP estimate applied in $A$ gives

$$
h_{-} \leq C_{n} \operatorname{diam}(A)\|g\|_{L^{n}(A)} \leq C_{n} K \delta_{2} \text { in } A
$$

and

$$
h_{+} \geq-C_{n} \operatorname{diam}(A)\|g\|_{L^{n}(A)} \geq-C_{n} K \delta_{2} \text { in } A .
$$


By restricting $\varepsilon \leq\left(\frac{L}{\left(C_{n} K\right)^{\alpha}}\right)^{\frac{1}{1-\alpha}}$, we can assume that

$$
\delta_{1}=(\varepsilon / L)^{1 / \alpha} \leq \frac{\varepsilon}{C_{n} K} .
$$

Then, for $\delta_{2} \leq \delta_{1}$, we have $C_{n} K \delta_{2} \leq \varepsilon$ and thus, for all $x \in A$, we have

$$
|v(x)-v(0)| \leq 2 \varepsilon+C_{1}\left(\inf \left\{y_{n}: y \in \bar{\Omega} \cap \partial B_{\delta_{2}}(0)\right\}\right)^{-1} x_{n} .
$$

The uniform convexity of $\Omega$ gives

$$
\inf \left\{y_{n}: y \in \bar{\Omega} \cap \partial B_{\delta_{2}}(0)\right\} \geq C_{2}^{-1} \delta_{2}^{2} .
$$

Therefore, choosing $\delta_{2}=\delta_{1}$, we obtain

$$
|v(x)-v(0)| \leq 2 \varepsilon+C_{1}\left(\inf \left\{y_{n}: y \in \bar{\Omega} \cap \partial B_{\delta_{2}}(0)\right\}\right)^{-1} x_{n}=2 \varepsilon+\frac{2 C_{1} C_{2}}{\delta_{2}^{2}} x_{n} \text { in } A .
$$

As a consequence, we have just obtained the following inequality

$$
|v(x)-v(0)| \leq 2 \varepsilon+\frac{2 C_{1} C_{2}}{\delta_{2}^{2}}|x|=2 \varepsilon+2 C_{1} C_{2} L^{2 / \alpha} \varepsilon^{-2 / \alpha}|x|
$$

for all $x, \varepsilon$ satisfying the following conditions

$$
|x| \leq \delta_{1}(\varepsilon):=(\varepsilon / L)^{1 / \alpha}, \varepsilon \leq\left(\frac{L}{\left(C_{n} K\right)^{\alpha}}\right)^{\frac{1}{1-\alpha}}:=c_{1}(\alpha, L, K, n) .
$$

Finally, let us choose

$$
\varepsilon=|x|^{\frac{\alpha}{\alpha+2}} .
$$

It satisfies the conditions in (2.5) if $c_{1} \geq|x|^{\frac{\alpha}{\alpha+2}} \geq L|x|^{\alpha}$, or

$$
|x| \leq \min \left\{c_{1}^{\frac{\alpha+2}{\alpha}}, L^{-\frac{\alpha+2}{\alpha^{2}}}\right\}:=\delta .
$$

Then, by (2.4), we have for all $x \in \Omega \cap B_{\delta}(0)$

$$
|v(x)-v(0)| \leq C|x|^{\frac{\alpha}{\alpha+2}}
$$

where $C=2+2 C_{1} C_{2} L^{2 / \alpha}$.

The above proposition, applied to $a_{i j}=U^{i j}$ where $U=\left(U^{i j}\right)$ is the matrix of cofactors of the Hessian matrix $D^{2} u$ of the convex function $u$ satisfying

$$
\lambda \leq \operatorname{det} D^{2} u \leq \Lambda
$$

gives the boundary continuity for solutions to the linearized Monge-Ampère equation

$$
U^{i j} v_{i j}=g .
$$

This combined with the interior Hölder continuity estimates of Caffarelli-Gutiérrez [CG] gives the global Hölder estimates for solutions to the linearized Monge-Ampère equations on uniformly convex domains. The proof follows the same lines as in the proofs of Theorem 2. 5 and Proposition 2.6 in [LS1]. The rest of this section will be devoted to the proof of these global Hölder estimates. 
2.2. The Localization Theorem. In this subsection, we state the main tool used in the proof of Theorem 1.4, the localization theorem.

Let $\Omega \subset \mathbb{R}^{n}$ be a bounded convex set with

$$
B_{\rho}\left(\rho e_{n}\right) \subset \Omega \subset\left\{x_{n} \geq 0\right\} \cap B_{\frac{1}{\rho}},
$$

for some small $\rho>0$. Assume that

(2.7) $\Omega$ contains an interior ball of radius $\rho$ tangent to $\partial \Omega$ at each point on $\partial \Omega \cap B_{\rho}$.

Let $u: \bar{\Omega} \rightarrow \mathbb{R}, u \in C^{0,1}(\bar{\Omega}) \cap C^{2}(\Omega)$ be a convex function satisfying

$$
\operatorname{det} D^{2} u=f, \quad 0<\lambda \leq f \leq \Lambda \quad \text { in } \Omega \text {, }
$$

and assume that

$$
u(0)=0, \quad \nabla u(0)=0 .
$$

Let $S_{h}(u)$ be the section of $u$ at 0 with level $h$ :

$$
S_{h}:=\{x \in \bar{\Omega}: \quad u(x)<h\} .
$$

If the boundary data has quadratic growth near $\left\{x_{n}=0\right\}$ then, as $h \rightarrow 0, S_{h}$ is equivalent to a half-ellipsoid centered at 0 . This is the content of the Localization Theorem proved in [S, S2]. Precisely, this theorem reads as follows.

Theorem 2.2 (Localization Theorem [S, S2]). Assume that $\Omega$ satisfies (2.6) - 2.7) and $u$ satisfies (2.8), (2.9) above and,

$$
\rho|x|^{2} \leq u(x) \leq \rho^{-1}|x|^{2} \quad \text { on } \partial \Omega \cap\left\{x_{n} \leq \rho\right\} .
$$

Then, for each $h<k$ there exists an ellipsoid $E_{h}$ of volume $\omega_{n} h^{n / 2}$ such that

$$
k E_{h} \cap \bar{\Omega} \subset S_{h} \subset k^{-1} E_{h} \cap \bar{\Omega} .
$$

Moreover, the ellipsoid $E_{h}$ is obtained from the ball of radius $h^{1 / 2}$ by a linear transformation $A_{h}^{-1}$ (sliding along the $x_{n}=0$ plane)

$$
\begin{gathered}
A_{h} E_{h}=h^{1 / 2} B_{1}, \quad \operatorname{det} A_{h}=1, \\
A_{h}(x)=x-\tau_{h} x_{n}, \quad \tau_{h}=\left(\tau_{1}, \tau_{2}, \ldots, \tau_{n-1}, 0\right),
\end{gathered}
$$

with

$$
\left|\tau_{h}\right| \leq k^{-1}|\log h|
$$

The constant $k$ above depends only on $\rho, \lambda, \Lambda, n$.

The ellipsoid $E_{h}$, or equivalently the linear map $A_{h}$, provides useful information about the behavior of $u$ near the origin. From Theorem 2.2 we also control the shape of sections that are tangent to $\partial \Omega$ at the origin. Before we state this result we introduce the notation for the section of $u$ centered at $x \in \bar{\Omega}$ at height $h$ :

$$
S_{x, h}(u):=\{y \in \bar{\Omega}: u(y)<u(x)+\nabla u(x)(y-x)+h\} .
$$


Proposition 2.3. Let $u$ and $\Omega$ satisfy the hypotheses of the Localization Theorem 2.0 at the origin. Assume that for some $y \in \Omega$ the section $S_{y, h} \subset \Omega$ is tangent to $\partial \Omega$ at 0 for some $h \leq c$ with $c$ universal. Then there exists a small constant $k_{0}>0$ depending on $\lambda$, $\Lambda, \rho$ and $n$ such that

$$
\begin{aligned}
& \nabla u(y)=a e_{n} \quad \text { for some } a \in\left[k_{0} h^{1 / 2}, k_{0}^{-1} h^{1 / 2}\right], \\
& k_{0} E_{h} \subset S_{y, h}-y \subset k_{0}^{-1} E_{h}, \quad k_{0} h^{1 / 2} \leq \operatorname{dist}(y, \partial \Omega) \leq k_{0}^{-1} h^{-1 / 2},
\end{aligned}
$$

with $E_{h}$ the ellipsoid defined in the Localization Theorem 2.2.

Proposition 2.3 is a consequence of Theorem 2.2 and was proved [S3].

2.3. Quadratic separation on the boundary. The quadratic separation from tangent planes on the boundary for solutions to the Monge-Ampère equation is a crucial assumption in the Localization Theorem 2.2. This is the case for $u$ in Theorem 1.4 as proved in [S2, Proposition 3.2].

Proposition 2.4. Let $u$ be as in Theorem 1.4. Then, on $\partial \Omega$, u separates quadratically from its tangent planes on $\partial \Omega$. This means that if $x_{0} \in \partial \Omega$ then

$$
\rho\left|x-x_{0}\right|^{2} \leq u(x)-u\left(x_{0}\right)-\nabla u\left(x_{0}\right)\left(x-x_{0}\right) \leq \rho^{-1}\left|x-x_{0}\right|^{2},
$$

for all $x \in \partial \Omega$, for some small constant $\rho$ universal.

When $x_{0} \in \partial \Omega$, the term $\nabla u\left(x_{0}\right)$ is understood in the sense that

$$
x_{n+1}=u\left(x_{0}\right)+\nabla u\left(x_{0}\right) \cdot\left(x-x_{0}\right)
$$

is a supporting hyperplane for the graph of $u$ but for any $\varepsilon>0$,

$$
x_{n+1}=u\left(x_{0}\right)+\left(\nabla u\left(x_{0}\right)-\varepsilon \nu_{x_{0}}\right) \cdot\left(x-x_{0}\right)
$$

is not a supporting hyperplane, where $\nu_{x_{0}}$ denotes the exterior unit normal to $\partial \Omega$ at $x_{0}$. In fact we show in [LS1, Proposition 4.1] that our hypotheses imply that $u$ is always differentiable at $x_{0}$ and then $\nabla u\left(x_{0}\right)$ is defined also in the classical sense.

2.4. Global Hölder continuity for solutions of the linearized Monge-Ampère equation. We are now ready to prove Theorem 1.4.

Proof of Theorem 1.4. We recall from Proposition 2.4 that $u$ separates quadratically from its tangent planes on $\partial \Omega$. Therefore, Proposition 2.3 applies. Let $y \in \Omega$ with

$$
r:=\operatorname{dist}(y, \partial \Omega) \leq c,
$$

for $c$ universal, and consider the maximal section $S_{y, \bar{h}(y)}$ centered at $y$, i.e.,

$$
\bar{h}(y)=\max \left\{h \mid \quad S_{y, h} \subset \Omega\right\} .
$$

When it is clear from the context, we write $\bar{h}$ for $\bar{h}(y)$. By Proposition 2.3 applied at the point

we have

$$
x_{0} \in \partial S_{y, \bar{h}} \cap \partial \Omega,
$$

$$
\bar{h}^{1 / 2} \sim r
$$


and $S_{y, \bar{h}}$ is equivalent to an ellipsoid $E$ i.e

$$
c E \subset S_{y, \bar{h}}-y \subset C E,
$$

where

$$
E:=\bar{h}^{1 / 2} A_{\bar{h}}^{-1} B_{1}, \quad \text { with } \quad\left\|A_{\bar{h}}\right\|,\left\|A_{\bar{h}}^{-1}\right\| \leq C|\log \bar{h}| ; \operatorname{det} A_{\bar{h}}=1
$$

We denote

$$
u_{y}:=u-u(y)-\nabla u(y)(x-y) .
$$

The rescaling $\tilde{u}: \tilde{S}_{1} \rightarrow \mathbb{R}$ of $u$

$$
\tilde{u}(\tilde{x}):=\frac{1}{\bar{h}} u_{y}(T \tilde{x}) \quad x=T \tilde{x}:=y+\bar{h}^{1 / 2} A_{\bar{h}}^{-1} \tilde{x},
$$

satisfies

$$
\operatorname{det} D^{2} \tilde{u}(\tilde{x})=\tilde{f}(\tilde{x}):=f(T \tilde{x})
$$

and

$$
B_{c} \subset \tilde{S}_{1} \subset B_{C}, \quad \tilde{S}_{1}=\bar{h}^{-1 / 2} A_{\bar{h}}\left(S_{y, \bar{h}}-y\right),
$$

where $\tilde{S}_{1}$ represents the section of $\tilde{u}$ at the origin at height 1 .

We define also the rescaling $\tilde{v}$ for $v$

$$
\tilde{v}(\tilde{x}):=v(T \tilde{x})-v\left(x_{0}\right), \quad \tilde{x} \in \tilde{S}_{1} .
$$

Then $\tilde{v}$ solves

$$
\tilde{U}^{i j} \tilde{v}_{i j}=\tilde{g}(\tilde{x}):=\bar{h} g(T \tilde{x}) .
$$

Now, we apply Caffarelli-Gutiérrez's interior Hölder estimates [CG, TW3] to $\tilde{v}$ to obtain

$$
\left|\tilde{v}\left(\tilde{z}_{1}\right)-\tilde{v}\left(\tilde{z}_{2}\right)\right| \leq C\left|\tilde{z}_{1}-\tilde{z}_{2}\right|^{\beta}\left\{\|\tilde{v}\|_{L^{\infty}\left(\tilde{S}_{1}\right)}+\|\tilde{g}\|_{L^{n}\left(\tilde{S}_{1}\right)}\right\}, \quad \forall \tilde{z}_{1}, \tilde{z}_{2} \in \tilde{S}_{1 / 2},
$$

for some small constant $\beta \in(0,1)$ depending only on $n, \lambda, \Lambda$.

By (2.14), we can decrease $\beta$ if necessary and thus we can assume that

$$
2 \beta \leq \frac{\alpha}{\alpha+2}:=2 \gamma
$$

Note that, by (2.13)

$$
\|\tilde{g}\|_{L^{n}\left(\tilde{S}_{1}\right)}=\bar{h}^{1 / 2}\|g\|_{L^{n}\left(S_{y, \bar{h}}\right)} .
$$

We observe that (2.12) and (2.13) give

$$
B_{C r|\log r|}(y) \supset S_{y, \bar{h}} \supset S_{y, \bar{h} / 2} \supset B_{c \frac{r}{\mid l o g r} \mid}(y)
$$

and

$$
\operatorname{diam}\left(S_{y, \bar{h}}\right) \leq C r|\log r|
$$

By Proposition 2.1, we have

$$
\|\tilde{v}\|_{L^{\infty}\left(\tilde{S}_{1}\right)} \leq C \operatorname{diam}\left(S_{y, \bar{h}}\right)^{2 \gamma} \leq C(r|\log r|)^{2 \gamma} .
$$

Hence

$$
\left|\tilde{v}\left(\tilde{z}_{1}\right)-\tilde{v}\left(\tilde{z}_{2}\right)\right| \leq C\left|\tilde{z}_{1}-\tilde{z}_{2}\right|^{\beta}\left\{(r|\log r|)^{2 \gamma}+\bar{h}^{1 / 2}\|g\|_{L^{n}\left(S_{y, \bar{h}}\right)}\right\} \forall \tilde{z}_{1}, \tilde{z}_{2} \in \tilde{S}_{1 / 2}
$$


Rescaling back and using

$$
\tilde{z}_{1}-\tilde{z}_{2}=\bar{h}^{-1 / 2} A_{\bar{h}}\left(z_{1}-z_{2}\right)
$$

and the fact that

$$
\left|\tilde{z}_{1}-\tilde{z}_{2}\right| \leq \| \bar{h}^{-1 / 2} A_{\bar{h}}||\left|z_{1}-z_{2}\right| \leq C \bar{h}^{-1 / 2}|\log \bar{h}|\left|z_{1}-z_{2}\right| \leq C r^{-1}|\log r|\left|z_{1}-z_{2}\right|,
$$

we find

$$
\left|v\left(z_{1}\right)-v\left(z_{2}\right)\right| \leq\left|z_{1}-z_{2}\right|^{\beta} \quad \forall z_{1}, z_{2} \in S_{y, \bar{h} / 2} .
$$

Notice that this inequality holds also in the Euclidean ball $B_{c \frac{r}{|l o g r|}}(y) \subset S_{y, \bar{h} / 2}$. Combining this with Proposition 2.1, we easily obtain that

$$
[v]_{C^{\beta}(\bar{\Omega})} \leq C,
$$

for some $\beta \in(0,1), C$ universal.

For completeness, we include the details. By rescaling the domain, we can assume that

$$
\Omega \subset B_{1 / 100}(0) .
$$

We estimate, for $x$ and $y$ in $\Omega$

$$
\frac{|v(x)-v(y)|}{|x-y|^{\beta}} .
$$

Let $r_{x}=\operatorname{dist}(x, \partial \Omega)$ and $r_{y}=\operatorname{dist}(y, \partial \Omega)$. Suppose that $r_{y} \leq r_{x}$, say, and take $x_{0} \in \partial \Omega$ and $y_{0} \in \partial \Omega$ such that $r_{x}=\left|x-x_{0}\right|$ and $r_{y}=\left|y-y_{0}\right|$. From the interior Hölder estimates of Caffarelli-Gutiérrez, we only need to consider the case $r_{y} \leq r_{x} \leq c$.

Assume first that

$$
|x-y| \leq c \frac{r_{x}}{\left|\log r_{x}\right|} .
$$

Then $y \in B_{c \frac{r_{x}}{\left|l o g r_{x}\right|}}(x) \subset S_{x, \bar{h}(x) / 2}$. By (2.15), we have

$$
\frac{|v(x)-v(y)|}{|x-y|^{\beta}} \leq 1 .
$$

Assume finally that

We claim that

$$
|x-y| \geq c \frac{r_{x}}{\left|\log r_{x}\right|} .
$$

Indeed, if

$$
r_{x} \leq C|x-y||\log | x-y||
$$

$$
1>r_{x} \geq|x-y||\log | x-y|| \geq|x-y|
$$

then

$$
r_{x} \leq \frac{1}{c}|x-y|\left|\log r_{x}\right| \leq \frac{1}{c}|x-y||\log | x-y|| .
$$

Now, we have

$$
\left|x_{0}-y_{0}\right| \leq r_{x}+|x-y|+r_{y} \leq C|x-y||\log | x-y|| .
$$


Hence, by Proposition 2.1 and recalling $2 \gamma=\frac{\alpha}{\alpha+2}$,

$$
\begin{aligned}
|v(x)-v(y)| & \leq\left|v(x)-v\left(x_{0}\right)\right|+\left|v\left(x_{0}\right)-v\left(y_{0}\right)\right|+\left|v\left(y_{0}\right)-v(y)\right| \\
& \leq C\left(r_{x}^{2 \gamma}+\left|x_{0}-y_{0}\right|^{\alpha}+r_{y}^{2 \gamma}\right) \\
& \leq C(|x-y||\log | x-y||)^{2 \gamma} \leq C|x-y|^{\beta} .
\end{aligned}
$$

\section{Global second Derivative estimates for the PRESCRibed AfFine mean CURVATURE EQUATION}

In this section, we prove Theorem [1.2, Let $u \in C^{4}(\bar{\Omega})$ be the uniformly convex solution of (1.1) -(1.3). We first establish bounds on the determinant $\operatorname{det} D^{2} u$ via those of $w$.

Lemma 3.1. There exists a constant $C>0$ depending only on $n, p, \theta, \Omega,\|f\|_{L^{p}(\Omega)},\|\psi\|_{W^{2, p}(\Omega)}$, and $\inf _{\Omega} \psi$ such that any uniformly convex solution $u \in C^{4}(\bar{\Omega})$ of (1.1)-(1.3) satisfies

$$
C^{-1} \leq w \leq C .
$$

Proof. Because $f \leq 0$, by the maximum principle, $w$ attains its minimum on the boundary. Since $\inf _{\Omega} \psi>0$, we obtain the first inequality in (3.1).

For the upper bound of $w$, we use (1.2) in the form $\operatorname{det} D^{2} u=w^{\frac{1}{\theta-1}}$ and the AleksandrovBakelman-Pucci estimate. By this estimate, we have

$$
\begin{aligned}
\|w\|_{L^{\infty}(\Omega)} & \leq\|\psi\|_{L^{\infty}(\partial \Omega)}+C\left\|\frac{f}{(\operatorname{det} U)^{1 / n}}\right\|_{L^{n}(\Omega)} \\
& =\|\psi\|_{L^{\infty}(\partial \Omega)}+C\left\|\frac{f}{\left(\operatorname{det} D^{2} u\right)^{\frac{n-1}{n}}}\right\|_{L^{n}(\Omega)} \\
& =\|\psi\|_{L^{\infty}(\partial \Omega)}+C\left\|f w^{\frac{n-1}{n(1-\theta)}}\right\|_{L^{n}(\Omega)} \\
& \leq\|\psi\|_{L^{\infty}(\partial \Omega)}+C\|w\|_{L^{\infty}(\Omega)}^{\frac{n-1}{n(1-\theta)}}\|f\|_{L^{n}(\Omega)} .
\end{aligned}
$$

Because $\theta<1 / n$, we have

$$
\frac{n-1}{n(1-\theta)}<1
$$

and the second inequality in (3.1) follows.

We are now ready to complete the proof of Theorem 1.2,

Proof of theorem 1.2. By Lemma 3.1.

$$
C^{-1} \leq \operatorname{det} D^{2} u \leq C
$$


Note that, by (1.1), $w$ is the solution to the linearized Monge-Ampère equation $U^{i j} w_{i j}=f$ with boundary data $w=\psi$. Because $\psi \in W^{2, p}(\Omega)$ with $p>n, \psi$ is clearly Hölder continuous on $\partial \Omega$. Thus, by Theorem 1.4, $w$ is Hölder continuous up the boundary. Rewriting (1.2) as

$$
\operatorname{det} D^{2} u=w^{\frac{1}{\theta-1}}
$$

and noticing $u=\varphi$ on $\partial \Omega$ where $\varphi \in C^{3}(\bar{\Omega})$, we obtain $u \in C^{2, \alpha}(\bar{\Omega})$ [TW2, Theorem 1.1]. Thus (1.1) is a uniformly elliptic, second order partial differential equations in $w$. Hence $w \in W^{2, p}(\Omega)$ and in turn $u \in W^{4, p}(\Omega)$ with desired estimate

$$
\|u\|_{W^{4, p}(\Omega)} \leq C,
$$

where $C$ depends on $n, p, \theta, \partial \Omega, \Omega,\|f\|_{L^{p}(\Omega)},\|\varphi\|_{W^{4, p}(\Omega)},\|\psi\|_{W^{2, p}(\Omega)}$, and $\inf _{\Omega} \psi$.

\section{REFERENCES}

[Ab] Abreu, M. Kähler geometry of toric varieties and extremal metrics, Inter. J. Math. 9 (1998), 641651.

[Bl] Blaschke, W. Vorlesungen über Differential geometrie, Berlin, 1923.

[CC] Caffarelli, L. A., and Cabré, X. Fully nonlinear elliptic equations. American Mathematical Society Colloquium Publications, volume 43, 1995.

[C2] Caffarelli, L. A. Interior $W^{2, p}$ estimates for solutions of Monge-Ampère equation, Ann. of Math. 131 (1990), 135-150.

[CG] Caffarelli, L. A.; Gutiérrez, C. E. Properties of the solutions of the linearized Monge-Ampère equation. Amer. J. Math. 119 (1997), no. 2, 423-465.

[D1] Donaldson, S. K. Scalar curvature and stability of toric varieties. J. Differential Geom. 62 (2002), no. 2, 289-349.

[D2] Donaldson, S. K. Interior estimates for solutions of Abreu's equation. Collect. Math. 56 (2005), no. $2,103-142$

[D3] Donaldson, S. K. Extremal metrics on toric surfaces: a continuity method. J. Differential Geom. 79 (2008), no. 3, 389-432.

[D4] Donaldson, S. K. Constant scalar curvature metrics on toric surfaces. Geom. Funct. Anal. 19 (2009), no. $1,83-136$.

[GT] Gilbarg, D.; Trudinger, N. S. Elliptic partial differential equations of second order. Reprint of the 1998 edition. Classics in Mathematics. Springer-Verlag, Berlin, 2001.

[GN1] Gutiérrez, C.; Nguyen, T. Interior gradient estimates for solutions to the linearized Monge-Ampère equations, Adv. Math. 228 (2011), 2034-2070.

[LS1] Le, N. Q.; Savin, O. Boundary regularity for solutions to the linearized Monge-Ampère equations, Preprint, arXiv:1109.5677v1 [math.AP].

[NS] Nomizu, K. and Sasaki, T. Affine differential geometry, Cambridge University Press, 1994

[S] Savin, O. A localization property at the boundary for the Monge-Ampère equation. arXiv:1010.1745v2 [math.AP].

[S2] Savin, O. Pointwise $C^{2, \alpha}$ estimates at the boundary for the Monge-Ampère equation. arXiv:1101.5436v1 [math.AP]

[S3] Savin, O. Global $W^{2, p}$ estimates for the Monge-Ampère equation. arXiv:1103.0456 v1 [math.AP].

[Si] Simon, U. Affine differential geometry, in Handbook of differential geometry, North-Holland, Amsterdam, 2000, 905-961.

[TW] Trudinger, N. S.; Wang, X. J. The Bernstein problem for affine maximal hypersurfaces. Invent. Math. 140 (2000), no. 2, 399-422. 
[TW1] Trudinger, N.S. and Wang, X.J., The affine plateau problem, J. Amer. Math. Soc. 18(2005), 253289.

[TW2] Trudinger N.S., Wang X.J, Boundary regularity for Monge-Ampère and affine maximal surface equations, Ann. of Math. 167 (2008), 993-1028.

[TW3] Trudinger, N. S.; Wang, X. J. The Monge-Ampère equation and its geometric applications. Handbook of geometric analysis. No. 1, 467-524, Adv. Lect. Math. (ALM), 7, Int. Press, Somerville, MA, 2008.

[Zh] Zhou, B. The first boundary value problem for Abreu's equation, preprint, arXiv:1009.1834v1 [math.AP].

Department of Mathematics, Columbia University, New York, Ny 10027, USA, and School of Engineering, Tan Tao University, Long An, Vietnam

E-mail address: namle@math.columbia.edu, nam.le@ttu.edu.vn 